\title{
Synthesis of dyes derived from 1-aryl-5-amino-4-cyanopyrazoles
}

\author{
Sanjay Gupta, Aravind Sivasubramanian, Lígia M. Rodrigues, Ana P. Esteves, \\ Radim Hrdina $^{\mathrm{a}}$ and Ana M.F. Oliveira-Campos ${ }^{1}$
}

Centro de Química, Universidade do Minho, Campus de Gualtar, 4710-057 Braga, Portugal

${ }^{a}$ Faculty of Chemical Technology, University of Pardubice, Czech Republic

\begin{abstract}
Eight dyes were prepared by diazotisation of substituted amino-cyano pyrazoles and coupling to anilines or 2-naphthol. The dyes were fully characterised by spectroscopic techniques. The cyano group of the pyrazoles was found to be susceptible to hydrolysis during preparation.
\end{abstract}

Keywords: cyanopyrazole; azo dyes; diazotisation.

\section{Introduction}

Heterocycles are extensively used in disperse dye chemistry for textile or non-textile applications including their application in reprography, functional dye and non-linear optical systems, photodynamic therapy and lasers [1].

Azo dyes containing heterocyclic rings lead to brighter and often deeper shades than their benzene analogues [2] and they are still very important for applications such as disperse dyes for polyester fibres [3].

\footnotetext{
${ }^{1}$ Corresponding author: Fax: +351253678983

E-mail address: amcampos@quimica.uminho.pt
} 
Following previous interest in our group for heterocyclic textile dyes [4] and also for pyrazole derivatives [5] for various purposes, it was decided to prepare dyes derived from arylpyrazoles, containing electron withdrawing groups, such as carboxyl and nitro.

\section{Results and Discussion}

The preparation of the pyrazoles involved the reaction of the required hydrazines with ethoxymethylenemalononitrile in ethanol at room temperature [6]. The 5-amino-4-cyano$N$-phenylpyrazoles 1 were obtained in 94\% (1b) and 43\% (1a) yields and were fully characterized.

The pyrazole containing the nitro group (1a) had previously been used as starting material for the preparation of dyes [6]. Aminocyanopyrazoles 1 were evidenced on IR by signals at 2215 to $2243 \mathrm{~cm}^{-1}$ and in the NMR spectra by a singlet for proton 3 (e.g. at $7.64 \mathrm{ppm}$ for compound 1a ) and the pair of doublets expected for the para substituted phenyl ring.

The preparation of the dyes was started by diazotization of the amino group on the pyrazole ring by the nitrosylsulfuric method. The coupling reaction was performed in dilute $\mathrm{H}_{2} \mathrm{SO}_{4}$ when $N$, $N$-diethylaniline and meta- $N, N$-diethylbenzanilide were the coupling components (Scheme 1). In three cases (3, 4 and $\mathbf{5})$, the cyano group was hydrolysed during the preparation of the dye to the corresponding amide, even when the nitrosylsulfuric acid was diluted with a mixture of acetic and propionic acids.

The coupling with 2-naphthol was always performed under alkaline conditions $(\mathrm{NaOH})$. For the sake of simplicity in the discussion, the dyes were organized on the basis of the coupling components. 


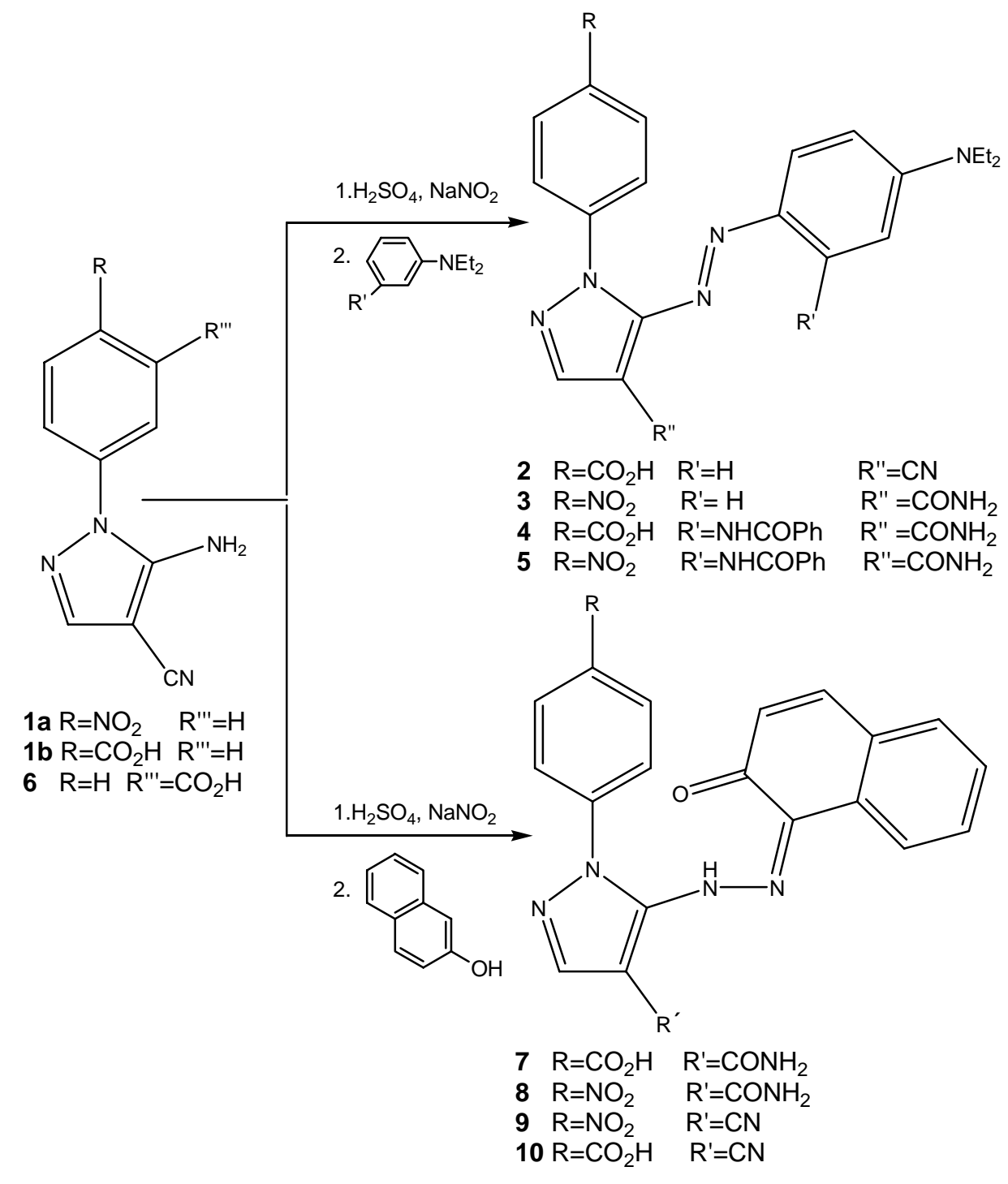

\section{Dyes obtained with Anilines}

When the respective diazotized pyrazoles were coupled either with $N, N$ - diethylaniline or with $m-N, N$-diethylbenzanilide, the reaction proceeded smoothly in $19 \%$ to $38 \%$ yields.

On perusal of the proton NMR spectra of dyes $(\mathbf{3}, \mathbf{4}$, and 5) apart from expected signals, two broad well separated singlets (e.g. $\delta 7.26$ and 7.91 in 4), accounting for one proton each, were found. Also in the ${ }^{13} \mathrm{C}$ NMR spectra, the resonance corresponding to the $\mathrm{CN}$ group $(\delta 114.64)$ at the position $\mathrm{C} 3$ of the pyrazole was found missing, and an additional carbonyl signal was found at $163.58 \mathrm{ppm}$. The IR spectra of the dye 4 showed no presence of the peak at $2243 \mathrm{~cm}^{-1}$ corresponding to the cyano group. 
Hence, on the basis of the spectroscopic data, it could be rationalized that the cyano group was susceptible to hydrolysis and the dyes were obtained as the corresponding amides. The only exception was observed with the dye 2, where the $\mathrm{CN}$ group remained intact.

The amount of $\mathrm{H}_{2} \mathrm{SO}_{4}$ used for the diazotization was varied and even under the optimized conditions (for both diazotization and coupling) the hitherto produced results were obtained. When the concentration of $\mathrm{H}_{2} \mathrm{SO}_{4}$ was significantly decreased, no diazotization occurred.

It is reported that the diazotization of amino-nitropyrazole can be performed under conditions [6], where after the formation of nitrosylsulphuric acid, the medium is diluted by the addition of acetic / propionic acids $(5: 1 \mathrm{v} / \mathrm{v})$ mixture. Even though the solubility of the pyrazoles 1a and $\mathbf{1 b}$ in that mixture was marginal, the experiments were carried out in a lower molar scale.

Even in this method, the cyano group (with the exception of dye 2) of the pyrazoles was found to be converted into the respective amides.

\section{Dyes obtained with 2-naphthol}

The diazotization of the pyrazoles for coupling with 2-naphthol was performed by both ways mentioned above (nitrosylsulfuric method with or without the addition of mixture of acetic and propionic acids $(5: 1 \mathrm{v} / \mathrm{v})$. The coupling proceeded in sodium hydroxide solution.

When the diazotization conditions did not involve dilution with the organic acids mixture, the cyano group was found hydrolysed to amide, in the resulting dyes $\mathbf{7 , 8}$. 
In our previous work of dyes from aminopyrazole 6 and 2-naphthol, nitrosylsulfuric method with the addition of mixture of acetic and propionic acids $(5: 1 \mathrm{v} / \mathrm{v})$ for diazotization, evolved an orange dye with the cyano group intact on the pyrazoles [4a].

Consequently it was decided to obtain dyes from aminopyrazoles (1a and 1b) by nitrosylsulfuric method with the addition of mixture of acetic and propionic acids $(5: 1$ $\mathrm{v} / \mathrm{v}$ ) in the conditions previously described. The preparations were successful and products isolated were the nitriles $\mathbf{9}$ and $\mathbf{1 0}$.

In the Table 1 yields, melting points, visible and IR absorption characteristics of the products are shown. All the compounds were characterized by spectroscopic methods and elemental analysis or high resolution mass spectrometry. Other techniques such as ${ }^{13} \mathrm{C}$ NMR, HMQC and HMBC were also used.

Table 1. Yields, melting points and UV/Visible and IR spectroscopic data for dyes 2-5 and 7-10

\begin{tabular}{|c|c|c|c|c|}
\hline Dye & $\begin{array}{c}\text { Yield } \\
{[\%]}\end{array}$ & $\begin{array}{l}\text { M.p. } \\
{\left[{ }^{\circ} \mathrm{C}\right]}\end{array}$ & $\begin{array}{c}\text { UV/VIS (in EtOH) } \\
\lambda_{\max }[\mathrm{nm}]\left(\varepsilon\left[\mathrm{dm}^{3} \cdot \mathrm{mol}^{-1} \cdot \mathrm{cm}^{-1}\right]\right)\end{array}$ & $\begin{array}{l}\operatorname{IR}(* *) \\
{\left[\mathrm{cm}^{-1}\right]}\end{array}$ \\
\hline 2 & $38^{*}$ & $249-250$ & $\begin{array}{l}543.5\left(3.4 \times 10^{4}\right) ; 396.0\left(2.1 \times 10^{3}\right) \\
304.0\left(7.9 \times 10^{3}\right) ; 283.0\left(6.8 \times 10^{3}\right) \\
246.5\left(1.7 \times 10^{4}\right)\end{array}$ & $\begin{array}{l}3463 ; 2453 ; 2249 ; 1723 ; 1677 ; 1658 \\
1607 ; 1418 ; 1327 ; 1288\end{array}$ \\
\hline 3 & $33^{*}$ & $264-266$ & $\begin{array}{l}465.0\left(3.9 \times 10^{3}\right) ; 401.5\left(2.4 \times 10^{3}\right) \\
306.5\left(1.7 \times 10^{4}\right) ; 253.5\left(1.0 \times 10^{4}\right)\end{array}$ & $\begin{array}{l}3464 ; 3120 ; 2996 ; 2938 ; 1693 ; 1595 ; \\
1522 ; 1496 ; 1337 ; 1276 ; 1177\end{array}$ \\
\hline 4 & $24^{*}$ & $298-299$ & $\begin{array}{l}512.5\left(1.6 \times 10^{4}\right) ; 381.0\left(1.9 \times 10^{3}\right) \\
318.0\left(5.0 \times 10^{3}\right) ; 298.0\left(4.1 \times 10^{3}\right) \\
253.5\left(1.4 \times 10^{4}\right)\end{array}$ & $\begin{array}{l}3425 ; 2977 ; 2925 ; 2237 ; 1694 ; 1682 ; \\
1620 ; 1556 ; 1536 ; 1393 ; 1367 ; 1336 ; \\
1268 ; 1214 ; 1173\end{array}$ \\
\hline 5 & $19 *$ & $257-259$ & $\begin{array}{l}522.5\left(3.3 \times 10^{4}\right) ; 398.0\left(4.6 \times 10^{3}\right) \\
267.0\left(1.7 \times 10^{4}\right)\end{array}$ & $\begin{array}{l}3406 ; 2977 ; 2938 ; 1706 ; 1664 ; 1595 ; \\
1553 ; 1521 ; 1498 ; 1469 ; 1406 ; 1332 ; \\
1288 ; 1267 ; 1167\end{array}$ \\
\hline 7 & 29 & $310-312$ & $470.0\left(8.3 \times 10^{3}\right)$ & $\begin{array}{l}3462 ; 1694 ; 1632 ; 1563 ; 1332 ; 1255 ; \\
1158 ; 1169\end{array}$ \\
\hline 8 & 23 & $>300$ & $\begin{array}{l}472.0\left(6.9 \times 10^{3}\right) \\
284.0\left(8.1 \times 10^{3}\right)\end{array}$ & $\begin{array}{l}3450 ; 1721 ; 1695 ; 1568 ; 1462 ; 1432 ; \\
1329 ; 1259 ; 1154 ; 1171\end{array}$ \\
\hline 9 & 21 & $252-254$ & $\begin{array}{l}541\left(1.6 \times 10^{4}\right) ; 339\left(1.58 \times 10^{4}\right) ; \\
292\left(1.4 \times 10^{4}\right)\end{array}$ & $\begin{array}{l}3460 ; 2736 ; 2671 ; 2229 ; 1710 ; \\
1613 ; 1594 ; 1249\end{array}$ \\
\hline 10 & 19 & $292-94$ & $\begin{array}{l}522.5\left(1.5 \times 10^{4}\right) ; 330\left(9.9 \times 10^{3}\right) ; \\
274\left(1.6 \times 10^{4}\right)\end{array}$ & $\begin{array}{l}3468 ; 2726 ; 2670 ; 2230 ; 1692 ; \\
1608 ; 1312 ; 1168\end{array}$ \\
\hline
\end{tabular}

* Yields for method A; for method B the values were comparable. ${ }^{\#}$ Dyes 9, 10 in DMF.

** $\mathrm{KBr}$ pellets for dyes 2-5; Nujol mull for dyes 7-10. 


\section{Conclusion}

Eight dyes were prepared, in low yields, by diazotisation of substituted aminocyanopyrazoles and coupling to anilines or 2-naphthol. It was observed that the cyano group on the pyrazole ring was labile under nitrosylsulfuric diazotization conditions. When this solution was diluted with a mixture of acetic and propionic acids $(5: 1 \mathrm{v} / \mathrm{v})$ and the coupling took place in base, as it was the case for the naphthol derivatives, the cyano group remained intact.

\section{Experimental}

\subsection{General}

Melting points are uncorrected, IR spectra were determined on a Perkin Elmer FTIR1600 and UV spectra were determined on a Hitachi U-2000. ${ }^{1} \mathrm{H}$ NMR spectra were recorded at $300 \mathrm{MHz}$ and ${ }^{13} \mathrm{C}$ NMR spectra were determined at $75.4 \mathrm{MHz}$ both on a Varian Unity Plus Spectrometer. Mass spectra were obtained by electron impact except for compounds $\mathbf{7}$ and $\mathbf{8}$ where $\mathrm{FAB}^{+}$was applied. High resolution mass spectra were obtained on a AutoSpec E spectrometer. Elemental analyses were obtained on a Leco CHNS-932 instrument. TLC was carried out on plates coated with silica gel $60 \mathrm{~F}_{254}$. Column chromatography was performed on silica gel $(<230$ mesh) with mixtures of light petroleum and ethyl acetate of increasing polarity, unless other conditions are described. Light petroleum refers to the fraction boiling in the range $40-60{ }^{\circ} \mathrm{C}$.

\subsection{General method of preparation of aminocyanopyrazoles}

The mixture of arylhydrazine $(33 \mathrm{mmol})$ and ethoxymethylenemalononitrile $(33 \mathrm{mmol})$ was stirred for 30 minutes and then ethanol $(20 \mathrm{~mL})$ was added. The mixture was stirred 
at room temperature for 24 hours. The precipitated product was filtered off and recrystallized from an appropriate solvent.

\subsubsection{5-Amino-1-(4’-nitrophenyl)-1H-pyrazole-4-carboxamide (1a)}

The title compound was obtained as a yellowish solid after re-crystallization from acetone-water (isolated yield $43 \%$ ), m.p. $=221.8-223.5^{\circ} \mathrm{C}$ (lit. m.p. $224-225^{\circ} \mathrm{C}[6,7]$ ). ${ }^{1} \mathrm{H}$ NMR (DMSO-d $\left.{ }_{6}\right) \delta(\mathrm{ppm}): 7.06\left(2 \mathrm{H}\right.$, br s, $\left.\mathrm{NH}_{2}\right), 7.83\left(2 \mathrm{H}, \mathrm{d}, J=9.0 \mathrm{~Hz}, \mathrm{H}-2^{\prime}\right.$ and H-6'), 7.90 (1H, s, H-3), 8.35 (2H, d, J=9.3 Hz, H-3' and H-5').

${ }^{13} \mathrm{C}$ NMR (DMSO-d $\left.\mathrm{d}_{6}\right) \delta(\mathrm{ppm}): 74.35(\mathrm{C}-4), 114.43(\mathrm{CN}), 124.22(\mathrm{C}-2$ ' and C-6'), 125.01 (C-3' and C-5'), 142.82 (C-4'), 143.10 (C-3), 145.74 (C-1'), 151.99 (C-5).

IR(Nujol mull) $\left(v_{\max } \mathrm{cm}^{-1}\right): 3445,3316,3230,3210,2230,2218$ (weaker, side band), $1650,1598$.

HRMS: Calculated for $\mathrm{C}_{10} \mathrm{H}_{7} \mathrm{~N}_{5} \mathrm{O}_{2}: 229.0600$; found: $\left(\mathrm{M}^{+}\right) 229.0603$.

\subsubsection{5-Amino-1-(4'-carboxyphenyl)-1H-pyrazole-4-carbonitrile (1b)}

It was obtained as an orange solid (yield $94 \%$ ), re-crystallized from ethanol/water (yield $89 \%$, m.p. $275-277^{\circ} \mathrm{C}$.

${ }^{1} \mathrm{H}$ NMR (DMSO-d $) \delta$ (ppm): $6.88\left(2 \mathrm{H}\right.$, br s, $\left.\mathrm{NH}_{2}\right), 7.65(2 \mathrm{H}, \mathrm{d}, J=8.7 \mathrm{~Hz}, \mathrm{H}-2$ ' and H6'), $7.84(1 \mathrm{H}, \mathrm{s}, \mathrm{H}-3), 8.06$ (2H, d, J=8.7 Hz, H-3' and H-5'), 13.15 (1H, br s, COOH).

${ }^{13} \mathrm{C}$ NMR (DMSO-d $\left.\mathrm{d}_{6}\right) \delta(\mathrm{ppm}): 72.94(\mathrm{C}-4), 114.64(\mathrm{CN}), 123.55\left(\mathrm{C}-2^{\prime}\right.$ and $\left.\mathrm{C}-6{ }^{\prime}\right)$, 129.61 (C-1'), 130.65 (C-3'and C-5'), 141.08 (C-4'), 142.44 (C-3), 151.58 (C-5), $166.64(\mathrm{C}=\mathrm{O})$.

IR(Nujol mull) $\left(v_{\max } \mathrm{cm}^{-1}\right): 3457,3297,3180,2243$ 1688, 1639, 1608, 1537.

Anal. calcd for $\mathrm{C}_{11} \mathrm{H}_{8} \mathrm{~N}_{4} \mathrm{O}_{2}$ : C, 57.89; H, 3.51; N, 24.56. Found: C, 58.16; H, 3.69; N, 24.44. 
HRMS: Calculated for $\mathrm{C}_{11} \mathrm{H}_{8} \mathrm{~N}_{4} \mathrm{O}_{2}: 228.0647$; found: $\left(\mathrm{M}^{+}\right)$228.0647.

\subsection{General method of preparation of dyes $\mathbf{2 - 5}$}

\section{Method A}

Sodium nitrite (2 mmoles) was dissolved in concentrated $\mathrm{H}_{2} \mathrm{SO}_{4}(4 \mathrm{~g})$ at room temperature followed by the addition of the aminocyano derivative ( 2 mmoles). The mixture was stirred for two hours at room temperature.

To a cooled solution $\left(0-5{ }^{\circ} \mathrm{C}\right)$ of $N, N$-diethylaniline derivative $(1.5$ mmoles $)$ in water (300 mL) and concentrated $\mathrm{H}_{2} \mathrm{SO}_{4}(2 \mathrm{~mL})$ was added dropwise, the previous diazonium solution added and the mixture was stirred for 30 minutes. The precipitated product was collected and purified by column chromatography and re-crystallization.

\section{Method B}

$\mathrm{NaNO}_{2}(35 \mathrm{mg}, 0.5 \mathrm{mmol})$ was added to concentrated $\mathrm{H}_{2} \mathrm{SO}_{4}(1.5 \mathrm{~mL})$ with external cooling (ice-acetone bath). The suspension was heated to $60^{\circ} \mathrm{C}$ and again cooled to 0 $5{ }^{\circ} \mathrm{C}$. To this cooled solution the mixture $(3 \mathrm{~mL})$ of acetic and propionic acids $(5: 1 \mathrm{v} / \mathrm{v})$ was added and it was stirred for 10 minutes. The pyrazole $(0.5 \mathrm{mmol})$ dissolved (or dispersed) in acetic and propionic acids $(5: 1 \mathrm{v} / \mathrm{v}, 3 \mathrm{~mL})$ was carefully added in portions and the mixture was stirred continuosly for 120 minutes with external cooling $\left(0-5^{\circ} \mathrm{C}\right)$. To a cooled solution of $N, N$-diethylaniline derivative $(1.5 \mathrm{mmoles})$ in acetic: propionic acids $(5: 1 \mathrm{v} / \mathrm{v}, 2 \mathrm{~mL})$ the prepared diazonium solution was drop wise added and the mixture was stirred for 30 minutes at reaction $\mathrm{pH} 7$ (neutralized with solid anhydrous sodium acetate). The precipitated product was collected and purified by column chromatography and re-crystallization. 


\subsubsection{4-[4'-Cyano-5'-(4"'-diethylamino-phenylazo)-pyrazol-1'-yl]-benzoic acid (2)}

Both methods afforded the title compound as a dark red solid.

${ }^{1} \mathrm{H}$ NMR (DMSO-d $\left.{ }_{6}\right) \delta(\mathrm{ppm}): 13.70\left(1 \mathrm{H}\right.$, br s, OH); $8.20\left(1 \mathrm{H}, \mathrm{s}, \mathrm{H}-3^{\prime}\right) ; 8.10(2 \mathrm{H}, \mathrm{d}$, $J=8.4 \mathrm{~Hz}, \mathrm{H}-2$ and H-6); $7.81(2 \mathrm{H}, \mathrm{d}, J=8.7 \mathrm{~Hz}, \mathrm{H}-3$ and $\mathrm{H}-5) ; 7.66(2 \mathrm{H}, \mathrm{d}, J=9.6 \mathrm{~Hz}$, H-2" and H-6"'); $6.92\left(2 \mathrm{H}, \mathrm{d}, J=9.6 \mathrm{~Hz}, \mathrm{H}-3^{\prime \prime}\right.$ and $\left.\mathrm{H}-5^{\prime \prime}\right) ; 3.52(4 \mathrm{H}, \mathrm{q}, J=7.2 \mathrm{~Hz}, 2 \mathrm{x}$ $\left.\mathrm{CH}_{2}\right) ; 1.16\left(6 \mathrm{H}, \mathrm{t}, J=6.9 \mathrm{~Hz}, 2 \times \mathrm{CH}_{3}\right)$.

${ }^{13} \mathrm{C}$ NMR (DMSO-d $) \delta(\mathrm{ppm}): 12.55\left(2 \mathrm{x} \quad \mathrm{CH}_{3}\right) ; 44.70\left(2 \mathrm{xCH}_{2}\right) ; 105.14(\mathrm{CN}) ; 112.43$ (C-3"' and C-5"'); 124.97 (C-3 and C-5): 126.84 (C-2"' and C-6" '); 130.07 (C-2 and C6); $140.46(\mathrm{C}-\mathrm{N}=\mathrm{N}) ; 133.24$ (C-1); 141.74 (C-4); 144.23 (C-3'); 150.72 (C-5'); 152.97(C-4' $) ; 121.77\left(\mathrm{C}-4^{\prime}\right) ; 166.66(\mathrm{C}=\mathrm{O})$.

HRMS: Calculated for $\mathrm{C}_{21} \mathrm{H}_{20} \mathrm{O}_{2} \mathrm{~N}_{6}$ : 388.1648; found 388.1631.

4.3.2. 5-(4"-Diethylamino-phenylazo)-1-(4'-nitro-phenyl)-1H-pyrazole-4-carboxamide (3)

A dark red solid was obtained by both methods.

${ }^{1} \mathrm{H} \mathrm{NMR}\left(\mathrm{CDCl}_{3}\right) \delta(\mathrm{ppm}): 9.73(1 \mathrm{H}, \mathrm{br}, \mathrm{CON}-\mathrm{H}) ; 8.38(2 \mathrm{H}, \mathrm{dd}, J=7.1$ and $1.8 \mathrm{~Hz}, \mathrm{H}-$ $3^{\prime}$ and $\left.\mathrm{H}-5^{\prime}\right) ; 8.41(1 \mathrm{H}, \mathrm{s}, \mathrm{H}-3) ; 7.90\left(2 \mathrm{H}, \mathrm{dd}, J=6.9\right.$ and $2.1 \mathrm{~Hz}, \mathrm{H}-2^{\prime}$ and $\left.\mathrm{H}-6^{\prime}\right) ; 7.67$ $\left(2 \mathrm{H}, \mathrm{dd}, J=7.2\right.$ and $1.8 \mathrm{~Hz}, \mathrm{H}-2^{\prime \prime}$ and $\left.\mathrm{H}-6^{\prime \prime}\right) ; 6.74\left(2 \mathrm{H}, \mathrm{dd}, J=7.2\right.$ and $1.8 \mathrm{~Hz}, \mathrm{H}-3^{\prime \prime}$ and H-5" ); $5.99\left(1 \mathrm{H}\right.$, br s, CON-H); $3.52\left(4 \mathrm{H}, \mathrm{d}, J=9.6 \mathrm{~Hz}, 2 \times \mathrm{CH}_{2}\right) ; 1.28(6 \mathrm{H}, \mathrm{t}, J=6.9 \mathrm{~Hz}$, $\left.2 \times \mathrm{CH}_{3}\right)$.

${ }^{13} \mathrm{C} \mathrm{NMR}\left(\mathrm{CDCl}_{3}\right) \delta(\mathrm{ppm}): 12.63\left(\mathrm{CH}_{3}\right) ; 45.20\left(\mathrm{CH}_{2}\right) ; 107.83(\mathrm{C}-4) ; 111.78(\mathrm{C}-3$ " and C-5' '); 124.05 (C-3' and C-5'); 126.13 (C-2' and C-6'); 126.69 (C-2' 'and C-6' '); 142.24 (C-1"'); 144.41 (C-1'); 145.85 (C-3); 146.42 (C-4'); 149.07 (C-5); 152.32 (C-4' ); 162. $44(\mathrm{C}=\mathrm{O}$ amide $)$. 
MS-EI (m/z, \%) $408.15\left(\mathrm{M}^{+}+1,8\right) ; 258.05$ (100). The molecule was unstable under EI conditions and it was only possible to obtain HRMS for the base peak. Calculated for $\mathrm{C}_{10} \mathrm{H}_{7} \mathrm{~N}_{6} \mathrm{O}_{3}$ 259.0580. Found: 259.0522 (corresponds to elimination of $N, N$ diethylaniline from the molecular ion).

4.4.3. 4-[5'-(2"-Benzoylamino-4"'-diethylamino-phenylazo)-4'-carbamoyl-pyrazol-1'yl]-benzoic acid (4)

The title compound was obtained as a dark red solid by both methods.

${ }^{1} \mathrm{H}$ NMR $\left(\mathrm{DMSO}_{-} \mathrm{d}_{6}\right) \delta(\mathrm{ppm}): 13.70(1 \mathrm{H}$, very br s, OH); $10.50(1 \mathrm{H}, \mathrm{s}, \mathrm{NH}) ; 8.13(1 \mathrm{H}$, s, H-3'); $8.02\left(1 \mathrm{H}, \mathrm{d}, J=2.7 \mathrm{~Hz}, \mathrm{H}-3^{\prime \prime}\right.$ '); 7.94 (2H, dd, $J=1.8$ and $8.7 \mathrm{~Hz}, \mathrm{H}-2$ and H-6); $7.91(1 \mathrm{H}$, br s, NH); $7.80(2 \mathrm{H}, \mathrm{d}, J=7.0 \mathrm{~Hz}$, ortho-Phe); $7.69(2 \mathrm{H}, \mathrm{dd}, J=2.1$ and $8.7 \mathrm{~Hz}$, H-3 and H-5); $7.61\left(1 \mathrm{H}, \mathrm{d}, J=9.6 \mathrm{~Hz}, \mathrm{H}-6{ }^{\prime \prime}\right) ; 7.58$ (1H, t, J=7.2 Hz, para-Phe); 7.49 (2H, t, J=7.2 Hz, meta-Phe); $7.26(1 \mathrm{H}, \mathrm{s}, \mathrm{NH}) ; 6.69\left(1 \mathrm{H}, \mathrm{dd}, J=2.7\right.$ and $9.6 \mathrm{~Hz}, \mathrm{H}-5^{\prime}$ '); $3.52\left(4 \mathrm{H}, \mathrm{q}, J=6.9 \mathrm{~Hz}, 2 \mathrm{xCH}_{2}\right) ; 1.20\left(6 \mathrm{H}, \mathrm{t}, J=6.9 \mathrm{~Hz}, 2 \mathrm{CH}_{3}\right)$.

${ }^{13} \mathrm{C}$ NMR (DMSO-d 6 ) $\delta(\mathrm{ppm}): 12.64\left(2 \times \mathrm{CH}_{3}\right) ; 44.84\left(2 \times \mathrm{CH}_{2}\right) ; 100.57\left(\mathrm{C}-3{ }^{\prime \prime}\right) ;$ $108.34\left(\mathrm{C}-4^{\prime}\right) ; 108.65$ (C-5'”); 121.67 (C-6’'); 124.37 (C-3 and C-5): 127.26 (2C-oPhe); 128.86 (2C-m-Phe); 129.52 (C-1); 130.12 (C-2 and C-6); 131.52 (C-1'’); 132.09 (C-p-Phe); 133.85 (C-1' '); 140.25 (C-4'); 142.21 (C-3'); 142.44 (C-4); 150.72 (C-5'); $152.97\left(\mathrm{C}-2^{\prime \prime}\right) ; 163.58\left(\mathrm{C}=\mathrm{ONH}_{2}\right) ; 165.24(\mathrm{C}=\mathrm{O}-\mathrm{Phe}) ; 166.53(\mathrm{C}=\mathrm{OOH})$.

Anal. calcd for: $\mathrm{C}_{28} \mathrm{H}_{27} \mathrm{~N}_{7} \mathrm{O}_{4} .1 / 2 \mathrm{H}_{2} \mathrm{O}: \mathrm{C}, 62.91 ; \mathrm{H}, 5.28 ; \mathrm{N}, 18.34$. Found: $\mathrm{C}, 62.46 ; \mathrm{H}$, $5.23 ; \mathrm{N}, 17.93$.

HRMS: Calculated for $\mathrm{C}_{28} \mathrm{H}_{27} \mathrm{O}_{4} \mathrm{~N}_{7}: 525.2125$; found 525.2150. 
4.4.4. 5-(2"'-Benzoylamino-4"'-diethylamino-phenylazo)-1-(4'-nitro-phenyl)-1Hpyrazole -4- carboxamide (5)

The title compound was obtained as a dark red solid, by methods A and B.

${ }^{1} \mathrm{H}$ NMR (DMSO-d 6 ) $\delta$ (ppm): $10.60(1 \mathrm{H}, \mathrm{s}, \mathrm{NH}) ; 8.17(1 \mathrm{H}, \mathrm{s}, \mathrm{H}-3) ; 8.16(2 \mathrm{H}, \mathrm{d}, J=9.3$ Hz, H-3'and H-5'); 8.05 (1H, d, J=2.4 Hz, H-3'”); 7.95 (1H, br s, NH); 7.86 (2H, d, $J=9.0 \mathrm{~Hz}, \mathrm{H}-2^{\prime}$ and H-6' $)$; 7.77 (2H, d, J=7.2 Hz, ortho-Phe); 7.66 (1H, d, J=9.6 Hz, H6’’); $7.55(1 \mathrm{H}, \mathrm{t}, J=7.5 \mathrm{~Hz}$, para-Phe); 7.45 (2H, t, J=7.5 Hz, meta-Phe); $7.31(1 \mathrm{H}, \mathrm{s}$, $\mathrm{NH}) ; 6.72\left(1 \mathrm{H}, \mathrm{dd}, J=2.7\right.$ and $9.6 \mathrm{~Hz}, \mathrm{H}-5$ ' '); $3.53\left(4 \mathrm{H}, \mathrm{q}, J=6.9 \mathrm{~Hz}, 2 \times \mathrm{CH}_{2}\right) ; 1.21(6 \mathrm{H}$, t, $\left.J=7.2 \mathrm{~Hz}, 2 \times \mathrm{CH}_{3}\right)$.

${ }^{13} \mathrm{C}$ NMR (DMSO-d 6$) \delta(\mathrm{ppm}): 12.63\left(2 \times \mathrm{CH}_{3}\right) ; 44.90\left(2 \times \mathrm{CH}_{2}\right) ; 100.54\left(\mathrm{C}-3{ }^{\prime \prime}\right)$; 108.76 (C-4); 108.80 (C-5'’); 123.09 (C-6’'); 124.48 (C-3'and C-5'): 124.86 (C-2' and C-6'); 127.15 (2C-o-Phe); 128.80 (2C-m-Phe); 131.41 (C-1’'); 131.99 (C-p-Phe); 133.87 (C-1"'); 140.10 (C-2' ); 143.12 (C-3); 143.83 (C-1'); 145.48 (C-4'); 150.83 (C5); $153.18\left(\mathrm{C}-4^{\prime \prime}\right) ; 163.37\left(\mathrm{C}=\mathrm{ONH}_{2}\right) ; 165.32(\mathrm{C}=\mathrm{O}-\mathrm{Phe})$.

Anal. calcd for $\mathrm{C}_{27} \mathrm{H}_{26} \mathrm{~N}_{8} \mathrm{O}_{4} \cdot \mathrm{H}_{2} \mathrm{O}:$ C, 59.55; H, 5.18; N, 20.58. Found: C, 60.09; H, $5.04 ; \mathrm{N}, 20.03$.

HRMS: Calculated for $\mathrm{C}_{27} \mathrm{H}_{26} \mathrm{O}_{4} \mathrm{~N}_{8}$ : 526.2077; found 526.2099.

\subsection{Method of preparation of dyes 7 and $\mathbf{8}$}

$\mathrm{NaNO}_{2}(35 \mathrm{mg}, 0.5 \mathrm{mmol})$ was added to concentrated $\mathrm{H}_{2} \mathrm{SO}_{4}(5 \mathrm{~mL})$ with external cooling (ice-acetone bath) the suspension was stirred for $10-15$ minutes at $20{ }^{\circ} \mathrm{C}$ and again cooled to $0-5^{\circ} \mathrm{C}$. To this cooled solution the pyrazole $(0.5 \mathrm{mmol})$ was carefully added in portions and the mixture was stirred continuously for 120 minutes with external cooling. 
The coupling component, 2-naphthol (72 $\mathrm{mg}, 0.5 \mathrm{mmol}$ ), was dissolved in $2 \mathrm{~mL}$ water and $\mathrm{NaOH}(20 \mathrm{mg}, 0.5 \mathrm{mmol})$ and the solution was externally cooled. To this the diazonium solution was dropwise added, keeping the temperature at $5{ }^{\circ} \mathrm{C}$ and reaction $\mathrm{pH}$ at $10(5 \mathrm{~N} \mathrm{NaOH}$ was added when necessary). The solution was either neutralised to $\mathrm{pH} 7$ (for compound 7) or was stirred continuously at room temperature (for compound 8). A precipitate which came out was filtered off, washed and dried.

4.5.1. 4-\{4'-Carbamoyl-5'-[N'-(2"'-oxo-2H-naphthalen-1"'-ylidene)-hydrazino]pyrazol-1'-yl\}-benzoic acid (7)

Re-crystallization from acetone yielded the orange dye 7 .

${ }^{1} \mathrm{H}$ NMR (acetone- $\left.\mathrm{d}_{6}\right) \delta(\mathrm{ppm}): 15.49(1 \mathrm{H}, \mathrm{s}, \mathrm{NH}-\mathrm{N}) ; 13.20\left(1 \mathrm{H}, \mathrm{br} \mathrm{s}, \mathrm{CO}_{2} \mathrm{H}\right) ; 8.26(1 \mathrm{H}$, s, H-3'); 8.07 (2H, dd, $J=6.6$ and $1.8 \mathrm{~Hz}, \mathrm{H}-2$ and H-6); $8.01(1 \mathrm{H}$, br s, CO-NH); 7.82 $\left(1 \mathrm{H}, \mathrm{d}, J=9.6 \mathrm{~Hz}, \mathrm{H}-4^{\prime \prime}\right) ; 7.65(2 \mathrm{H}, \mathrm{dd}, J=6.9$ and $1.8 \mathrm{~Hz}, \mathrm{H}-3$ and $\mathrm{H}-5) ; 7.59(1 \mathrm{H}, \mathrm{d}$, $J=7.2 \mathrm{~Hz}, \mathrm{H}-5^{\prime \prime}$ ); 7.43 (1H,br s, CO-NH); 7.31 (1H, pt, $J=7.5$ and 1.2 Hz, H-6" '); 6.94 $\left(1 \mathrm{H}, \mathrm{pt}, J=7.8\right.$ and $\left.1.2 \mathrm{~Hz}, \mathrm{H}-7^{\prime \prime}\right) ; 6.68\left(1 \mathrm{H}, \mathrm{d}, J=9.6 \mathrm{~Hz}, \mathrm{H}-3^{\prime \prime}\right) ; 6.36(1 \mathrm{H}, \mathrm{br} \mathrm{d}, J=8.1$ $\left.\mathrm{Hz}, \mathrm{H}-8^{\prime \prime}\right)$.

${ }^{13}$ C NMR (DMSO-d 6 ) $\delta(\mathrm{ppm}): 105.69\left(\mathrm{C}-4^{\prime}\right) ; 121.77$ (C-8' $)$; 130.58 (C-1); 130.42 (C2 and C-6); 130.25 (C-4a'"); 125.58 (C3'); 125.93 (C-3 and C-5); 127.05 (C-6"');

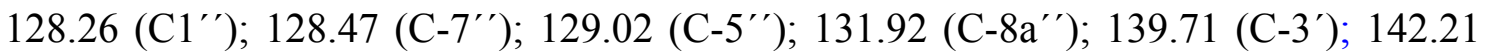
$\left(\mathrm{C}^{\prime \prime}{ }^{\prime}\right) ; 143.84\left(\mathrm{C}-5^{\prime}\right) ; 144.38(\mathrm{C}-4) ; 175.49\left(\mathrm{C}-2^{\prime \prime}\right) ; 166.70\left(\mathrm{CO}_{2} \mathrm{H}\right) ; 163.79(\mathrm{C}=\mathrm{O}$ amide)

HRMS: Calculated for $\mathrm{C}_{21} \mathrm{H}_{16} \mathrm{~N}_{5} \mathrm{O}_{4}$ : 402.1202; found: $(\mathrm{M}+1)^{+} 402.1186$. 
4.5.2. 1-(4'-Nitro-phenyl)-5-[N'-(2"'-oxo-2H-naphthalen-1"'-ylidene)-hydrazino]-1Hpyrazole-4-carboxamide (8)

The precipitated solid was purified by column chromatography (eluted with $2 \%$ $\mathrm{MeOH}-\mathrm{CHCl}_{3}$ ) and the dye $\mathbf{8}$ was obtained as an orange solid.

${ }^{1} \mathrm{H}$ NMR (DMSO-d 6 ) $\delta$ (ppm): $15.50(1 \mathrm{H}, \mathrm{s}, \mathrm{NH}-\mathrm{N}) ; 8.36(2 \mathrm{H}, \mathrm{dd}, J=7.1$ and $2.1 \mathrm{~Hz}, \mathrm{H}-$ $3^{\prime}$ and $\left.\mathrm{H}-5^{\prime}\right) ; 8.30(1 \mathrm{H}, \mathrm{s}, \mathrm{H}-3) ; 8.01(1 \mathrm{H}$, br s, CO-NH); $7.85(2 \mathrm{H}, \mathrm{dd}, J=6.8$ and 2.1 $\mathrm{Hz}, \mathrm{H} 2{ }^{\prime}$ and $\left.\mathrm{H}-6^{\prime}\right) ; 7.83\left(1 \mathrm{H}, \mathrm{d}, J=9.3 \mathrm{~Hz}, \mathrm{H}-4^{\prime \prime}\right) ; 7.60\left(1 \mathrm{H}, \mathrm{d}, J=6.9 \mathrm{~Hz}, \mathrm{H}-5^{\prime \prime}\right)$ ) 7.46 $\left(1 \mathrm{H}\right.$, br s, CO-NH); $7.32\left(1 \mathrm{H}, \mathrm{td}, J=8.1\right.$ and $\left.1.2 \mathrm{~Hz}, \mathrm{H}-6^{\prime \prime}\right)$; $6.97(1 \mathrm{H}, \mathrm{td}, J=7.8$ and 1.2 $\left.\mathrm{Hz}, \mathrm{H}-7^{\prime \prime}\right) ; 6.67$ (1H, d, J =9.6Hz, H-3' '); 6.40 (1H, d, J=6.9 Hz, H-8' ').

${ }^{13}$ C NMR (DMSO-d 6 ) $\delta$ (ppm): 105.69 (C-4); 121.33 (C-8' '); 124.65 (C-3' and C-5'); $125.67\left(\mathrm{C}-3^{\prime \prime}\right) ; 126.91\left(\mathrm{C}-2^{\prime}\right.$ and C-6'); $127.34\left(\mathrm{C}-6^{\prime \prime}\right) ; 128.37\left(\mathrm{C}-4 \mathrm{a}^{\prime \prime}\right) ; 128.63\left(\mathrm{C}-7^{\prime \prime}\right)$; 129.20 (C-5'); 130.39 (C-1"'); $131.80\left(\mathrm{C}-8 \mathrm{a}^{\prime \prime}\right) ; 140.22$ (C-3); 142.50 (C4'); 144.04 (C5); $145.91\left(\mathrm{C}^{\prime}{ }^{\prime}\right) ; 146.68\left(\mathrm{C}-4^{\prime}\right) ; 163.64\left(\mathrm{C}=\mathrm{O}\right.$ amide); $176.19\left(\mathrm{C}-2^{\prime \prime}\right)$.

HRMS: Calculated for $\mathrm{C}_{20} \mathrm{H}_{15} \mathrm{~N}_{6} \mathrm{O}_{4}: 403.1155$; found: $(\mathrm{M}+1)^{+} 403.1149$.

\subsection{Method of preparation of dyes $\mathbf{9}$ and $\mathbf{1 0}$}

$\mathrm{NaNO}_{2}(35 \mathrm{mg}, 0.5 \mathrm{mmol})$ was added to concentrated $\mathrm{H}_{2} \mathrm{SO}_{4}(1.5 \mathrm{~mL})$ with external cooling (ice-acetone bath). The suspension was heated to $60^{\circ} \mathrm{C}$ and again cooled to $0-$ $5{ }^{\circ} \mathrm{C}$. To this cooled solution the mixture $(3 \mathrm{~mL})$ of acetic and propionic acid $(5: 1 \mathrm{v} / \mathrm{v})$ was added and the reaction mixture was stirred for 10 minutes. The pyrazole $(0.5 \mathrm{mmol})$ in acetic and propionic acids $(5: 1 \mathrm{v} / \mathrm{v}, 3 \mathrm{~mL})$ was carefully added in portions and the mixture was stirred continuously for 120 minutes with external cooling.

The coupling was done as in the case of dyes 6 and 7. 
4.6.1. 1-(4'-Nitro-phenyl)-5-[N'-(2"'-oxo-2H-naphthalen-1"'-ylidene)-hydrazino]-1Hpyrazole-4-carbonitrile (9)

The precipitated solid was recrystallised from acetone.

${ }^{1} \mathrm{H} \mathrm{NMR}_{\left(\mathrm{DMSO}-\mathrm{d}_{6}\right)} \delta(\mathrm{ppm}): 13.65\left(1 \mathrm{H}, \mathrm{br}\right.$ s, NH-N); $8.67\left(1 \mathrm{H}, \mathrm{d}, J=8.1 \mathrm{~Hz}, \mathrm{H}-8^{\prime \prime}\right)$; $8.57(1 \mathrm{H}, \mathrm{s}, \mathrm{H}-3) ; 8.48\left(2 \mathrm{H}, \mathrm{dd}, J=7.2\right.$ and $2.1 \mathrm{~Hz}, \mathrm{H}-3^{\prime}$ and $\left.\mathrm{H}-5^{\prime}\right) ; 8.21(2 \mathrm{H}, \mathrm{dd}, J=6.9$ and $2.1 \mathrm{~Hz}, \mathrm{H}-2^{\prime}$ and $\left.\mathrm{H}-6^{\prime}\right) ; 8.14\left(1 \mathrm{H}, \mathrm{d}, J=9.3 \mathrm{~Hz}, \mathrm{H}-4^{\prime \prime}\right) ; 7.91(1 \mathrm{H}, \mathrm{d}, J=7.8 \mathrm{~Hz}, \mathrm{H}-$ $\left.5^{\prime \prime}\right) ; 7.67\left(1 \mathrm{H}, \mathrm{td}, J=6.9\right.$ and $\left.1.2 \mathrm{~Hz}, \mathrm{H}-7^{\prime \prime}\right) ; 7.53\left(1 \mathrm{H}, \mathrm{td}, J=6.9\right.$ and $\left.1.2 \mathrm{~Hz}, \mathrm{H}-6^{\prime \prime}\right) ; 7.14$ $\left(1 \mathrm{H}, \mathrm{d}, J=9.0 \mathrm{~Hz}, \mathrm{H}-3^{\prime \prime}\right)$.

${ }^{13}$ C NMR (DMSO-d $) \delta(p p m): 81.35(\mathrm{C}-4) ; 114.28(\mathrm{CN}) ; 120.89\left(\mathrm{C}-3^{\prime \prime}\right) ; 122.00$ (C$\left.8^{\prime \prime}\right) ; 125.03\left(\mathrm{C}-3^{\prime}\right.$ and $\left.\mathrm{C}-5^{\prime}\right) ; 125.89$ (C-2' and C-6'); 126.09 (C-6' $) ; 128.33\left(\mathrm{C}-4 \mathrm{a}^{\prime \prime}\right)$;

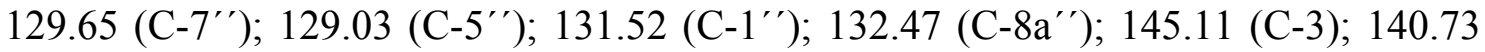
$\left(\mathrm{C} 4^{\prime \prime}\right) ; 153.26(\mathrm{C}-5) ; 142.27\left(\mathrm{C} 1^{\prime}\right) ; 147.10\left(\mathrm{C}-4^{\prime}\right) ; 158.66\left(\mathrm{C}-2^{\prime \prime}\right)$.

HRMS: Calculated for $\mathrm{C}_{20} \mathrm{H}_{12} \mathrm{O}_{3} \mathrm{~N}_{6}$ : 384.0971; found 384.0984.

4.6.2. 4-\{4'-Cyano-5'-[N'-(2"'-oxo-2H-naphthalen-1"'-ylidene)-hydrazino]-pyrazol-1'yl\}-benzoic acid (10)

Recrystallisation from ethanol yielded a dark brown orange dye.

${ }^{1} \mathrm{H}$ NMR $\left(\mathrm{DMSO}-\mathrm{d}_{6}\right) \delta(\mathrm{ppm}): 13.80(1 \mathrm{H}, \mathrm{br} \mathrm{s}, \mathrm{NH}) ; 9.44(1 \mathrm{H}, \mathrm{s}, \mathrm{COOH}) ; 8.67(1 \mathrm{H}, \mathrm{d}$, $\left.J=8.1 \mathrm{~Hz}, \mathrm{H}-8^{\prime \prime}\right) ; 8.54\left(1 \mathrm{H}, \mathrm{s}, \mathrm{H}-3^{\prime}\right) ; 8.18(2 \mathrm{H}, \mathrm{dd}, J=6.9$ and $1.8 \mathrm{~Hz}, \mathrm{H}-2$ and H-6); $8.15\left(1 \mathrm{H}, \mathrm{d}, J=9.0 \mathrm{~Hz}, \mathrm{H}-4^{\prime \prime}\right) ; 7.97(1 \mathrm{H}, \mathrm{dd}, J=7.2$ and $1.5 \mathrm{~Hz}, \mathrm{H}-3$ and $\mathrm{H}-5) ; 7.92(1 \mathrm{H}$, d, $\left.J=7.8 \mathrm{~Hz}, \mathrm{H}-5^{\prime \prime}\right) ; 7.67\left(1 \mathrm{H}, \mathrm{pt}, J=8.1\right.$ and $\left.1.2 \mathrm{~Hz}, \mathrm{H}-6^{\prime \prime}\right) ; 7.54(1 \mathrm{H}, \mathrm{pt}, J=8.1$ and 1.2 $\left.\mathrm{Hz}, \mathrm{H}-7^{\prime \prime}\right) ; 7.14\left(1 \mathrm{H}, \mathrm{d}, J=9.0 \mathrm{~Hz}, \mathrm{H}-3^{\prime \prime}\right)$.

${ }^{13}$ C NMR (DMSO-d $) \delta(\mathrm{ppm}): 81.29\left(\mathrm{C}-4^{\prime}\right) ; 114.21(\mathrm{CN}) ; 120.52\left(\mathrm{C}-3^{\prime \prime}\right) ; 121.98(\mathrm{C}-$ $\left.8^{\prime \prime}\right) ; 125.25$ (C-3 and C-5); 126.23 (C-6"'); 131.48 (C-4a' '); 129.07 (C-5' ); 129.71 (C- 


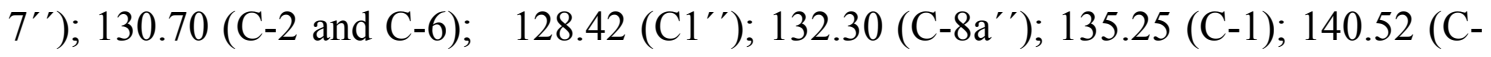

4); $140.84\left(\mathrm{C}-4^{\prime \prime}\right) ; 144.78\left(\mathrm{C}-3^{\prime}\right) ; 152.24\left(\mathrm{C}-5^{\prime}\right) ; 158.37\left(\mathrm{C}-2^{\prime \prime}\right) ; 166.38\left(\mathrm{CO}_{2} \mathrm{H}\right)$

HRMS: Calculated for $\mathrm{C}_{21} \mathrm{H}_{13} \mathrm{O}_{3} \mathrm{~N}_{5}: 383.1018$; found 383.1023 .

\section{Acknowledgements}

We thank Fundação para a Ciência e Tecnologia (FCT) and FEDER, for financial support and Miss Elisa Pinto for obtaining the NMR, and elemental analyses data.

The Post-Doctoral Grant (SFRH/BPD/20816/2004) for AS by FCT is also earnestly acknowledged.

R. Hrdina acknowledges the support of grant project CZ 351002 sponsored by The Ministry of Education, Youth and Sports of the Czech Republic.

\section{References}

[1] Towns AD. Dyes and Pigments 1999;42:3.

[2] Weaver MA, Shuttleworth L. Dyes and Pigments 1982;3:81.

[3] a) Singh K, Singh S, Taylor JA. Dyes and Pigments 2002;54:189. b) Singh K, Singh S, Mahajan A, Taylor JA. Color Technol 2003;119:198.

[4] a) Moura JCVP, Oliveira-Campos AMF, Griffiths J, Maia HLS, Gomes JINR. J Chem Research (S) 1995:128-9(M), 1995:924-937.

b) Miranda PC, Rodrigues LM, Gonçalves MST, Costa SPG, Hrdina R, OliveiraCampos AMF. Advances in Colour Science and Technology 2001;4(1): 21.

[5] Gonçalves MST, Oliveira-Campos AMF, Rodrigues LM, Proença MFRP, Griffiths J, Maia HLS, Kaja M, Hrdina R. J Chem Res 2004:115-117.

[6] Towne EB, Moore WH, Dickey JB. (to Eastman Kodak Co. ), CA 68:P 14072r, U.S. 3, 336, 285; 1967.

[7] Cheng CC, Robins RK. J Org Chem 1956;21:1240-56. 